\title{
The Emerging British Verticillium longisporum Population Consists of Aggressive Brassica Pathogens
}

\author{
Jasper R. L. Depotter, Luis Rodriguez-Moreno, Bart P. H. J. Thomma, ${ }^{\dagger}$ and Thomas A. Wood
}

First, second, and third authors: Laboratory of Phytopathology, Wageningen University and Research, Droevendaalsesteeg 1, 6708 PB Wageningen, The Netherlands; and first and fourth authors: Department of Crops and Agronomy, National Institute of Agricultural Botany, Huntingdon Road, CB3 OLE Cambridge, United Kingdom.

Accepted for publication 22 June 2017.

\begin{abstract}
Verticillium longisporum is an economically important fungal pathogen of brassicaceous crops that originated from at least three hybridization events between different Verticillium spp., leading to the hybrid lineages A1/D1, A1/D2, and A1/D3. Isolates of lineage A1/D1 generally cause stem striping on oilseed rape (Brassica napus), which has recently been reported for the first time to occur in the United Kingdom. Intriguingly, the emerging U.K. population is distinct from the north-central European stem striping population. Little is known about the pathogenicity of the newly emerged U.K. population; hence, pathogenicity tests were executed to compare British isolates to previously characterized reference strains. In addition to the model plant Arabidopsis thaliana, the pathogenicity of four British isolates was assessed on four cultivars of three Brassica crop species: oilseed rape (Quartz and Incentive), cauliflower

isolate infection were evaluated. The British isolates appeared to be remarkably aggressive, because plant biomass was significantly affected and severe vascular discoloration was observed. The British isolates were successful stem colonizers and the extent of fungal colonization negatively correlated with plant biomass of cauliflower and Quartz oilseed rape. However, in Quartz, the fungal colonization of A1/D1 isolates was significantly lower than that of the virulent reference isolate from lineage A1/D3, PD589. Moreover, despite levels of stem colonization similar to those of A1/D1 strains, PD589 did not cause significant disease on Incentive. Thus, A1/D1 isolates, including British isolates, are aggressive oilseed rape pathogens despite limited colonization levels in comparison with a virulent $\mathrm{A} 1 / \mathrm{D} 3$ isolate.
\end{abstract} (Clapton), and Chinese cabbage (Hilton). To this end, vascular discoloration of the roots, plant biomass accumulations, and fungal stem colonization upon
Additional keywords: Verticillium stem striping.
Verticillium fungi cause wilt diseases on hundreds of plant species, many of which are economically important crops. Verticillium dahliae is the most notorious member of this genus and can cause severe yield losses in crops such as olive and cotton (Fradin and Thomma 2006; Levin et al. 2003; Melero-Vara et al. 1995). Despite a wide host range that comprises hundreds of plant species, $V$. dahliae generally does not infect brassicaceous plants (Inderbitzin and Subbarao 2014; Zeise and von Tiedemann 2002). In contrast, V. longisporum is specialized on Brassicaceae family hosts, with oilseed rape as its most economically important target (Depotter et al. 2016a; Inderbitzin et al. 2011). In contrast to all other Verticillium spp., including $V$. dahliae, $V$. longisporum is not a haploid organism but rather an allodiploid as a consequence of interspecific hybridization. The species $V$. longisporum consists of three lineages, each representing a separate hybridization event. Four parental lines, including two V. dahliae isolates (D2 and D3), contributed to the different hybridization events. The two remaining parental lines represent two previously uncharacterized Verticillium spp. that have been provisionally called species A1 and species D1 (Inderbitzin et al. 2011). Species A1 participated in all three hybridization events and hybridized with D1, D2, and D3 to form the lineages A1/D1, A1/D2, and A1/D3, respectively. Conceivably, the allodiploid genome of $\mathrm{V}$. longisporum contributed to the host range shift, such that $V$. longisporum gained the capacity to infect species of Brassicaceae (Depotter et al. 2016b; Inderbitzin et al. 2011). The separate

${ }^{\dagger}$ Corresponding author: B. P. H. J. Thomma; E-mail: bart.thomma@wur.nl

B. P. H. J. Thomma and T. A. Wood contributed equally to this article.

*The $\boldsymbol{e}$-Xtra logo stands for "electronic extra" and indicates that five supplementary figures are published online.

(c) 2017 The American Phytopathological Society hybridization events seem to have affected the pathogenicity of V. longisporum lineages differently. Lineages A1/D1 and A1/D3 are found on various Brassicaceae species, whereas lineage A1/D2 is only known from horseradish in the United States (Inderbitzin et al. 2011; Yu et al. 2016). Furthermore, A1/D1 is the predominant lineage on oilseed rape and is also the most pathogenic $V$. longisporum lineage of this crop (Novakazi et al. 2015).

In addition to its different genetic constitution, V. longisporum is unique among Verticillium spp. for its disease symptom display on oilseed rape. Verticillium pathogens are xylem colonizers inducing occlusions in the vessels, which hampers the water transport in the xylem (Fradin and Thomma 2006). In response, plants with Verticillium infections generally develop wilting symptoms. However, these symptoms are lacking from $V$. longisporum infections on oilseed rape. Rather, black, unilateral stripes appear on the plant stem at the end of the growing season and, in a later stage, microsclerotia appear on the cortex under the stem epidermis (Heale and Karapapa 1999). Hence, the new common name "Verticillium stem striping" was coined to describe the $V$. longisporum disease on oilseed rape (Depotter et al. 2016a). Intriguingly, Verticillium stem striping symptoms fail to appear during pathogenicity tests when oilseed rape plants are grown under controlled conditions and seedlings are inoculated by dipping the roots in a spore suspension (Eynck et al. 2007; Eynck et al. 2009; Floerl et al. 2008; Zeise and von Tiedemann 2002). Under those conditions, plants exhibit chlorosis, vascular discoloration, and stunting at an early stage. The reasons for these differences in symptom development are currently unknown. Nevertheless, despite differences in disease symptomatology, it has previously been determined that root-dip pathogenicity tests in the glasshouse are a good proxy for oilseed rape cultivar resistance under field conditions (Knüfer et al. 2017).

In the past, $V$. dahliae and $V$. longisporum were considered a single species, and only at the end of the 1990s was V. longisporum proposed as a separate taxon (Karapapa et al. 1997). Hence, adequate characterization of V. longisporum strains in predating publications is hampered. However, 
on oilseed rape, Verticillium stem striping is exclusively caused by $V$. longisporum and, thus, trustworthy species assumptions can be made on this crop (Eynck et al. 2007). Verticillium stem striping was first reported in 1969 in the south of Sweden and became prevalent in northcentral Europe in the following years (Heale and Karapapa 1999; Kroeker 1970; Steventon et al. 2002; Zhou et al. 2006). Recently, the geographic range of Verticillium stem striping has expanded because oilseed rape production in Canada and the United Kingdom is now affected as well (CFIA 2015; Gladders et al. 2011). In the United Kingdom, Verticillium stem striping was reported for the first time in 2007 and is currently widespread (Gladders et al. 2011, 2013). Intriguingly, V. longisporum was most likely described earlier in the United Kingdom but on a different host: Brussels sprout (Isaac 1957). In congruence with other geographic regions, characterized $V$. longisporum strains from U.K. oilseed rape belong to the A1/D1 lineage (Depotter et al. in press). However, the U.K. population is distinct from the A1/D1 population in north-central Europe, where Verticillium stem striping is predominantly found (Depotter et al. in press). According to their relative geographic distribution in Europe, British isolates have been assigned to the "A1/D1 West" population, whereas German and Swedish A1/D1 isolates have been assigned to "A1/D1 East".

The recent emergence of Verticillium stem striping means that little is known about the pathogenicity of the British $V$. longisporum strains. Moreover, British strains belong to a distinct population from most other previously characterized $V$. longisporum strains isolated from oilseed rape. Hence, we performed pathogenicity tests to compare four British $V$. longisporum strains with five reference stains from different countries (Belgium, Germany, Japan, and United States), including all hybrid lineages. Pathogenicity was assessed thoroughly based on visual symptoms, biomass accumulation, and fungal colonization in the stem.

\section{MATERIALS AND METHODS}

Pathogenicity tests. Pathogenicity tests were conducted in order to compare the virulence of nine $V$. longisporum strains (Table 1). Three different Brassica crops were used: oilseed rape (Brassica napus var. oleifera), cauliflower (B. oleracea var. botrytis), and Chinese cabbage (B. rapa subsp. Pekinensis). Winter oilseed rape cultivars comprise open-pollinating and hybrid types, of which one of each was tested: 'Quartz' and 'Incentive', respectively. Furthermore, one Chinese cabbage cultivar ('Hilton'), and one cauliflower cultivar ('Clapton') were also included. Thus, in total, four Brassica cultivars were tested. Importantly, because of limited greenhouse space availability, pathogenicity tests on the different crops were not performed all at the same time. Plants were grown in climatecontrolled glasshouses under a cycle of $16 \mathrm{~h}$ of light and $8 \mathrm{~h}$ of darkness, with temperatures maintained between 20 and $28^{\circ} \mathrm{C}$ during the day and a minimum of $15^{\circ} \mathrm{C}$ overnight. Before sowing, seed were surface sterilized for $1 \mathrm{~min}$ in $70 \%$ ethanol followed by $15 \mathrm{~min}$ in $1 \%$ commercial bleach, then rinsed four times with sterile water. These sterilized seed were then sown in trays with sterile compost and kept in the glasshouse for 14 days. These 2-week-old seedlings were inoculated by dipping the roots for $30 \mathrm{~min}$ in a suspension of $1 \times 10^{6}$ conidiospores $\mathrm{ml}^{-1}$. Conidiospores were obtained from 3-week-old cultures grown on potato dextrose agar plates. In total, 15 plants were inoculated for every $V$. longisporum strain and 15 control plants were dipped in sterile water instead of a conidiospore suspension. Individual seedlings were planted in 9-cm-square pots with a 4:1:1 compost/sand/ loam mixture and pots were placed according to a random block design. The potting mixture had been autoclaved twice for $60 \mathrm{~min}$, with $24 \mathrm{~h}$ between each treatment. Plants were grown for 6 weeks before harvesting. Plants were harvested by cutting the stem just above the hypocotyls and pooled in five groups of three plants. For every pooled sample, the aboveground dry weight was subsequently determined by drying the samples at $100^{\circ} \mathrm{C}$ for a minimum of $12 \mathrm{~h}$. Furthermore, vascular discoloration of individual roots was scored at 6 weeks according to Tyvaert et al. (2014). A scale was used from 0 to 4 , where $0=$ no vascular discoloration and $1=1$ to $25,2=26$ to $50,3=51$ to 75 , and $4=76$ to $100 \%$ vascular discoloration of the root length. Pathogenicity tests were executed twice to confirm the virulence responses of the different strains. In addition to root vascular discoloration and dry weight, fungal stem colonization was evaluated. To this end, stems were removed at the hypocotyl and pooled in groups of three, and fungal colonization was assessed in the $1-\mathrm{cm}$ section adjacent to the hypocotyl. The remainders of the stems were used for dry weight determination. During the repeat experiment, Quartz plants were only grown until 25 days after inoculation to guarantee sufficient plant biomass from which to extract DNA because longer growth periods could lead to complete decay of the plants. Furthermore, in the second pathogenicity test, due to limited conidiospore availability, Chinese cabbage plants were inoculated with a water suspension of $5 \times 10^{5}$ conidiospores $\mathrm{ml}^{-1}$.

Arabidopsis thaliana (Col-0) plants were grown in a different glasshouse where the temperature was kept between 19 and $21^{\circ} \mathrm{C}$ and the same regime of light and darkness (16 and $8 \mathrm{~h}$, respectively) was used. Four plants of every treatment were grown. Plant inoculation occurred 3 weeks after sowing and roots were dipped for $10 \mathrm{~min}$ in a water suspension of $10^{6}$ conidiospores $\mathrm{ml}^{-1}$. Aboveground plant material was harvested for real-time polymerase chain reaction (PCR) analysis 3 weeks after inoculation.

Relative fungal quantification. Samples were taken for fungal biomass quantification and ground in liquid nitrogen. Approximately $200 \mathrm{mg}$ of ground plant material was dissolved in $500 \mu \mathrm{l}$ of cetyltrimethylammonium bromide (CTAB) buffer (55 mM CTAB, 0.1 M Tris [pH 8.0], $20 \mathrm{mM}$ EDTA [pH 8.0], $1.25 \mathrm{M} \mathrm{NaCl}$, and $0.25 \mathrm{mM}$ polyvinylpyrrolidone (PVP) 40). The buffer/plant extract was incubated for $30 \mathrm{~min}$ at $65^{\circ} \mathrm{C}$. Samples were centrifuged, supernatant was subsequently transferred to a clean tube, and $250 \mu \mathrm{l}$ of chloroform/isoamyl alcohol solution (24:1) was added. Samples were mixed thoroughly by inversion and subsequently centrifuged. Next, the supernatant was transferred into $50 \mu \mathrm{l}$ of ammonium acetate $(7.5 \mathrm{M})$ and $500 \mu \mathrm{l}$ of ethanol. After mixing, DNA was precipitated and the supernatant was removed. DNA pellets were washed twice with $70 \%$ ethanol and dissolved in DNase-free water.

TABLE 1. Verticillium longisporum isolate information

\begin{tabular}{|c|c|c|c|c|c|c|c|}
\hline Isolate & Region of origin & Country of origin & Original host & Year ${ }^{\mathrm{a}}$ & Lineage & MLG $^{\mathrm{b}}$ & Reference \\
\hline PD639 & Mecklenburg & Germany & Rape & 1990 & $\mathrm{~A} 1 / \mathrm{D} 1$ & MLG6 & Novakazi et al. 2015 \\
\hline $\mathrm{O} 1$ & Klein Brabant & Belgium & Cauliflower & $\ldots$ & A1/D1 & MLG23 & Tyvaert et al. 2014 \\
\hline PD356 & Illinois & United States & Horseradish & 1997 & A1/D2 & $\ldots$ & Novakazi et al. 2015 \\
\hline PD715 & Mecklenburg-Vorpommern & Germany & Rape & 1988 & A1/D3 & $\ldots$ & Novakazi et al. 2015 \\
\hline PD589 & Gunma & Japan & Cabbage & $\ldots$ & A1/D3 & $\ldots$ & Novakazi et al. 2015 \\
\hline VLB1 & Cambridgeshire & United Kingdom & Rape & 2014 & A1/D1 & MLG26 & Depotter et al. in press \\
\hline VLB2 & Leicestershire & United Kingdom & Rape & 2014 & A1/D1 & MLG15 & Depotter et al. in press \\
\hline VLB3 & Lincolnshire & United Kingdom & Rape & 2014 & A1/D1 & MLG26 & Depotter et al. in press \\
\hline VLB9 & Suffolk & United Kingdom & Rape & 2014 & $\mathrm{~A} 1 / \mathrm{D} 1$ & MLG17 & Depotter et al. in press \\
\hline
\end{tabular}

a Collection year.

b Multilocus genotype (MLG) of the A1/D1 isolates based on 12 polymorphic simple sequence repeat loci determined by Depotter et al. (in press).

c The allele of locus SSR25 could not be determined but all other loci resembled MLG26 (Depotter et al. in press). 
The amount of $V$. longisporum DNA in stems was quantified relatively to the amount of plant DNA by real-time PCR using the QuantStudio Flex Real-Time PCR System (Applied Biosystems, Foster City, CA). Fungal DNA was amplified with the V. longisporum-specific primer pair VITubF2/VITubR1 (GCAAAACCCTACCGGGTTATG/ AGATATCCATCGGACTGTTCGTA) (Debode et al. 2011) and the RuBisCO sequence targeting primer pair RubF/RubR (TATGCCTGCT TTGACCGAGA/AGCTACTCGGTTAGCTACGG) for plants. Realtime PCR was performed in reactions of $10 \mu$ l containing $500 \mathrm{nM}$ every primer and $5 \mu$ l of Power SYBR Green Master Mix (Applied Biosystems). The thermal program of the real-time PCR started with an initial denaturation step at $95^{\circ} \mathrm{C}$ for $10 \mathrm{~min}$, followed by 40 cycles of $15 \mathrm{~s}$ at $95^{\circ} \mathrm{C}$, $1 \mathrm{~min}$ at $62^{\circ} \mathrm{C}$, and $30 \mathrm{~s}$ at $72^{\circ} \mathrm{C}$. Specific amplification was verified running a melting curve: samples were heated to $95^{\circ} \mathrm{C}$ for $15 \mathrm{~s}$, cooled down to $60^{\circ} \mathrm{C}$ for $1 \mathrm{~min}$, and heated again to $95^{\circ} \mathrm{C}$ for $15 \mathrm{~s}$. Signals above 36 cycles are considered below the detection limit.

DNA of the Arabidopsis plants was isolated according to Fulton et al. (1995). Relative V. longisporum colonization was quantified according to Ellendorff et al. (2009) using the qPCR core kit (Eurogentec, Liège, Belgium). Real-time PCR was executed on an ABI7300 PCR System (Applied Biosystems) with the following thermal conditions: an initial $95^{\circ} \mathrm{C}$ denaturation step for $4 \mathrm{~min}$ and 30 cycles of denaturation for $15 \mathrm{~s}$ at $95^{\circ} \mathrm{C}$, annealing for $30 \mathrm{~s}$ at $60^{\circ} \mathrm{C}$, and extension for $30 \mathrm{~s}$ at $72^{\circ} \mathrm{C}$.

Data analysis. Significance levels were determined with the Mann-Whitney $U$ test (R Core Team 2015). Correlations were calculated with the Pearson correlation coefficient $(r)$. Data from the vascular discoloration consisted of too many ties for adequate $P$ value calculation. Hence, significant differences were calculated based on 5,000 bootstrap replicates of the median difference between random disease scores of two treatments.

\section{RESULTS}

The pathogenicity of four British $V$. longisporum isolates was compared with that of five previously characterized isolates, including isolates of all three $V$. longisporum hybridization lineages (Table 1). The four British isolates are adequate population representatives because their genotypes approximate the range of diversity within the British $V$. longisporum population (Table 1) (Depotter et al. in press). The isolates were tested on four different Brassica cultivars: two oilseed rape (Incentive and Quartz), one cauliflower (Clapton), and one Chinese cabbage (Hilton). Pathogenicity tests were repeated twice and similar pathogenicity outcomes for the different $V$. longisporum strains were obtained on both occasions. Pathogenic V. longisporum isolates stunted plant growth and leaves displayed chlorosis and necrosis (Supplementary Figs. S1, S2, S3, and S4). Moreover, the more aggressive isolates caused complete decay of Quartz plants within 6 weeks of inoculation. Furthermore, the pathogenicity of the British strains was also tested on the model plant $A$. thaliana (Col-0): VLB1, VLB2, and VLB9 induced leaf curling and necrosis (Supplementary Figs. S5). The same symptoms were observed for VLB3 but the plants were also heavily stunted, indicating that VLB3 is more aggressive than the other British strains on Arabidopsis.

The British $V$. longisporum isolates were pathogenic on all tested Brassica cultivars. Their vascular root tissues were discolored, which was generally significantly different from the control plants displaying no such symptoms (Fig. 1). Severe discoloration was similarly observed in the two A1/D1 reference strains. In contrast, the strain of lineage A1/D2, PD356, failed to induce vascular discoloration significantly different from the control treatment. Intriguingly, vascular discoloration varied for lineage A1/D3 depending on the strain and host. No significant root discoloration was observed in Incentive (Fig. 1B), whereas roots of Quartz, Clapton, and Hilton displayed discolorations ranging from nothing to severe (Fig. 1A, C, and D). Disease symptoms such as stunted growth and necrosis were in correspondence with the vascular discoloration of the roots and led to significant aboveground biomass reductions (Fig. 2). Here, reductions in biomass accumulations were among the highest for the British V. longisporum strains. Similarly, severe biomass reductions were observed for the two A1/D1 representatives (PD639 and O1), illustrating the high virulence of this lineage on Brassica hosts (Fig. 2). In contrast, PD356 was the least virulent V. longisporum strain, especially on oilseed rape, where no significant reductions in biomass accumulation were observed upon inoculation (Fig. 2A and B). PD356 inoculation significantly reduced the plant biomass of Clapton and Hilton but, nevertheless, it was one of the least aggressive isolates (Fig. 2C and D). Disease responses of the two A1/D3 representatives (PD715 and PD589) were, in contrast to the two A1/D1 representatives, dissimilar (Fig. 2). The German isolate PD715 was a weak pathogen unable to cause significant biomass reduction on Incentive (Fig. 2B) and was among the weakest strains tested on Quartz, Clapton, and Hilton (Fig. 2A, C, and D). In contrast, the Japanese A1/D3 isolate, PD589, strongly affected Brassica crops because PD589 was among the most severe isolates in Quartz, Clapton, and Hilton (Fig. 2A, C, and D). In contrast to the devastating outcome on Quartz oilseed, no significant biomass reduction was observed when Incentive was infected with PD589 (Fig. 2B).

To determine to what extent the symptomatology correlates with the amount of V. longisporum biomass inside the plant, V. longisporum DNA was quantified inside the stems relative to the amount of plant DNA. Verticillium spp. are xylem colonizers; hence, stem colonization is a good indication of strain aggressiveness. In correspondence with the observed disease symptoms, PD356 and PD715 were weak Brassica colonizers, because both strains were generally not detected in the stem, except for Hilton, where PD715 was detected in four of the five samples (Fig. 3). In contrast, all other V. longisporum isolates could be detected in most cases. In Quartz, PD589 was clearly the best colonizer, whereas the British and the A1/D1 strains were approximately present in equal levels (Fig. 3A). The colonization of the detected isolates was negatively correlated with the aboveground biomass of Quartz plants $(r=-0.6526$, $P=2.148 \times 10^{-5}$ ). In Incentive, significant differences in the colonization of the pathogenic isolates were observed, although these were not significantly correlated with plant biomass $(r=-0.2745, P=0.1105)$ (Fig. 3B). Intriguingly, although no significant biomass reduction was observed upon infection of PD589 on Incentive (Fig. 2B), PD589 was able to colonize the stem to a similar extent as the A1/D1 strains. In Clapton, high differences in colonization of the same isolates were observed between biological replicates (Fig. 3C). Similar to observations in Quartz, PD589 had the highest median colonization level, which was significantly higher than that of PD639 and VLB3. The fungal colonization of the detected isolates was also negatively correlated with the aboveground biomass of the cauliflower plants $(r=-0.4793$ $\left.P=4,127 \times 10^{-3}\right)$. Hilton was the only Brassica cultivar with detection for PD715; however, no differences in colonization between the isolates were observed (Fig. 3D). Similarly, PD715 was also detected in Arabidopsis for half of the inoculated plants and no significant differences in colonization were found between the treatments (Fig. 4).

\section{DISCUSSION}

The majority of emerging diseases originate from the introduction of pathogens in new geographic regions (Anderson et al. 2004). However, the recent outbreak of Verticillium stem striping in the United Kingdom originates from a previously established population (Depotter et al. in press). Similar to other newly emerging diseases, Verticillium stem striping it threatening for British oilseed rape, because the impact on yield is still relatively uncertain. In this study, the pathogenicity of four British V. longisporum isolates from four different counties was compared with five previously characterized strains, including strains from all $V$. longisporum lineages (Table 1). The British isolates were aggressive pathogens because they caused vascular discoloration and aerial biomass reduction, and were able to successfully colonize plant stem tissue (Figs. 1, 2, 3, and 4). The disease level because British isolates resembled those of the other two A1/D1 isolates (PD639 and O1) because plant colonization caused significant vascular discoloration and yield reductions on all Brassica crops (Figs. 1 and 2). This corresponds with a previous $V$. longisporum pathogenicity test that considered A1/ D1 as the most virulent $V$. longisporum lineage on oilseed rape and 
cauliflower (Novakazi et al. 2015). Thus, despite the genotypic differentiation, all A1/D1 West isolates (O1, VLB1, VLB2, VLB3, and VLB9) induced disease responses similar to those of A1/D1 East reference strain PD639 (Depotter et al. in press). However, further pathogenicity studies that include more strains and host species are needed to confirm that this is a general pattern for the two populations.
The unification of A1/D1 as a lineage of aggressive pathogens is in sheer contrast with the disease symptoms caused by reference strains from lineages A1/D2 and A1/D3. Lineage A1/D2 has hitherto only been found on horseradish and causes the severest disease on this crop of all V. longisporum strains (Novakazi et al. 2015; Yu et al. 2016). Isolate PD356 is a relatively poor colonizer of Brassica crops and caused no
A

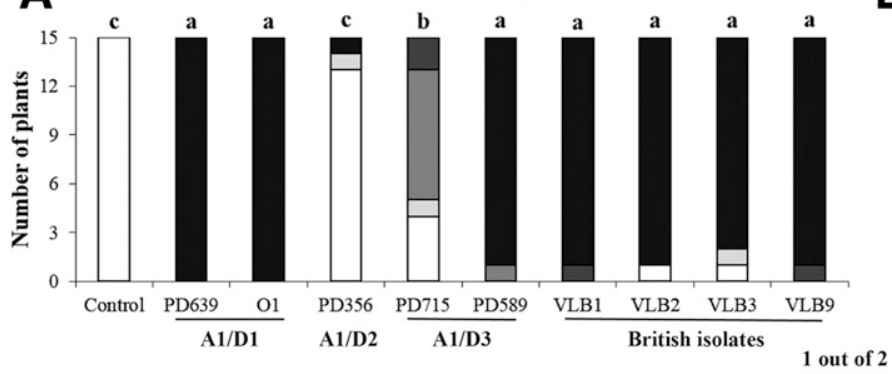

B

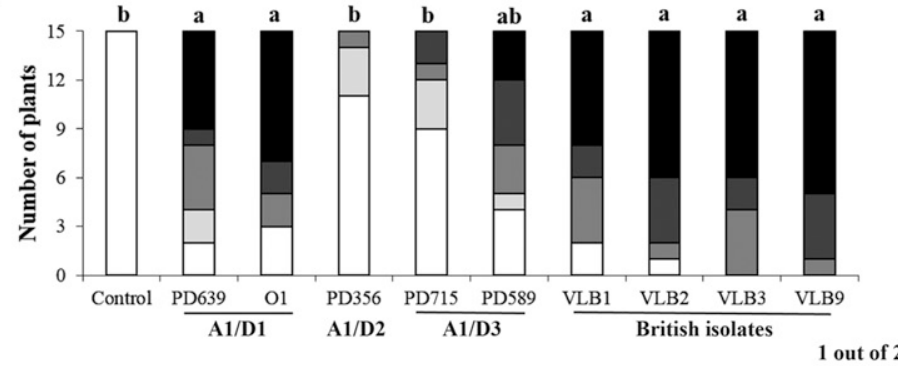

C

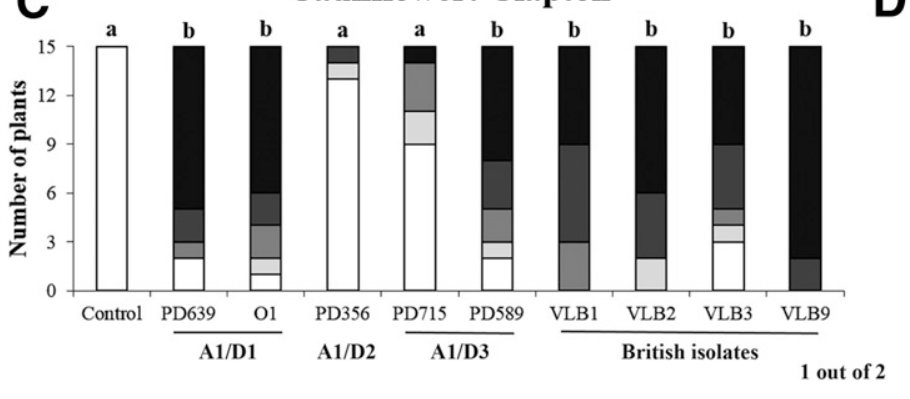

Cauliflower: Clapton

$\square=$ no discoloration $\square$

$=1-25 \%$ discoloration $\square$

$=26-50 \%$ discoloration

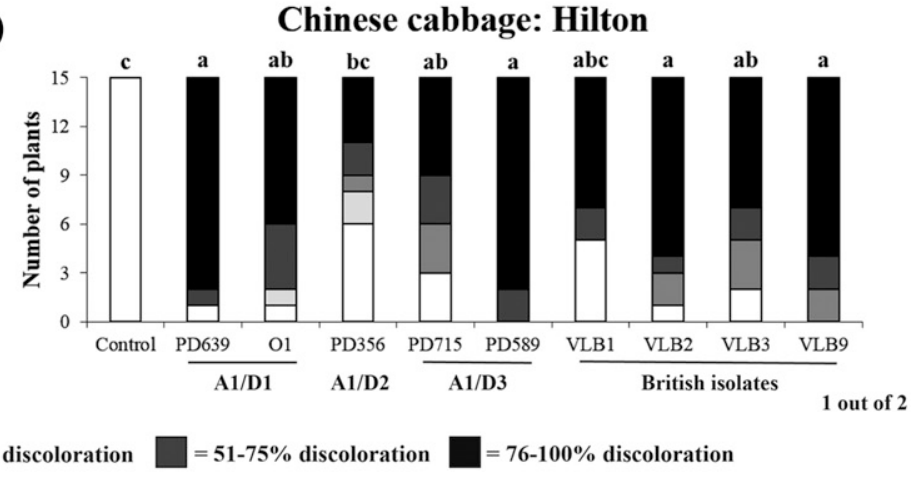

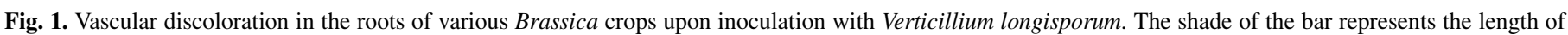

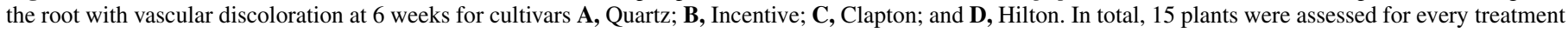

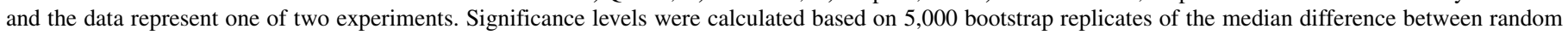
scores of two treatments $(P<0.05)$. Different letter labels indicate significant differences.

A

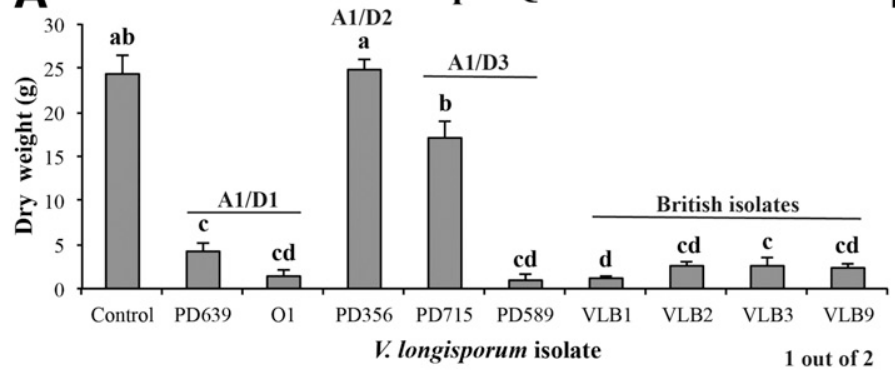

B

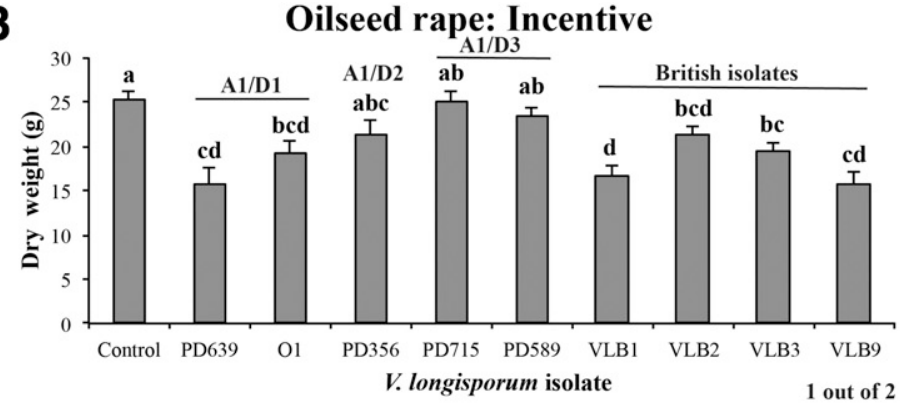

C

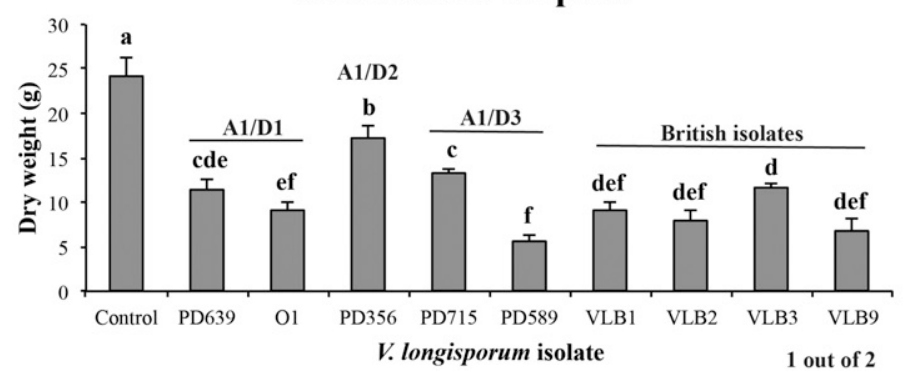

Cauliflower: Clapton

1 out of
D

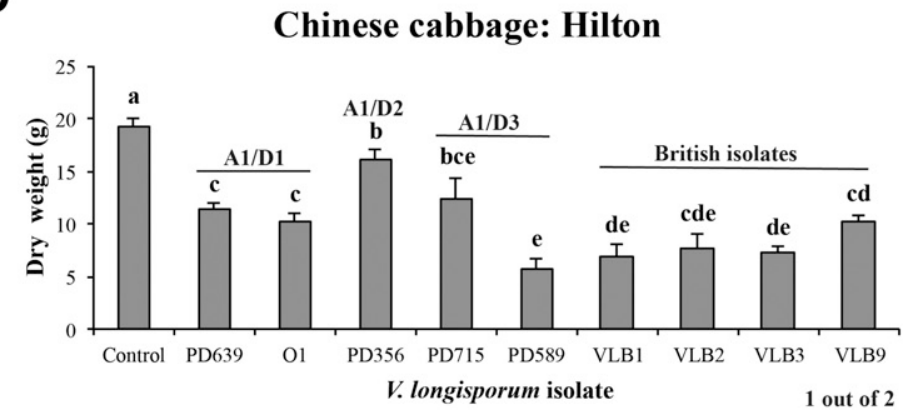

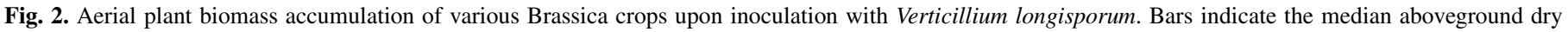

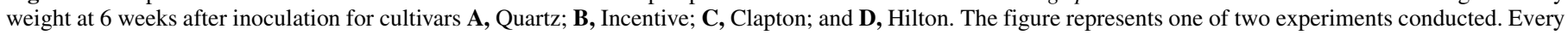

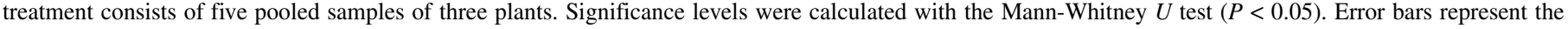
standard error. Different letter labels indicate significant differences. 
to little disease symptoms (Figs. 1, 2, and 3). Thus, the lack of disease in Brassica crops indicates that lineage A1/D2 is rather specialized on horseradish. However, A1/D2 came out as the most virulent $V$. longisporum lineage on cabbage (B. oleracea convar. capitata var. alba, 'Brunswijker') during pathogenicity tests of Novakazi et al. (2015), indicating that pathogenicity may vary within different varieties or crop subspecies. Previously, lineage A1/D3 was considered nonpathogenic on oilseed rape (Zeise and von Tiedemann 2002). However, a recent pathogenicity study identified a highly virulent strain on oilseed rape within lineage A1/D3: isolate PD589 (Novakazi et al. 2015). Accordingly, in this study, lineage A1/D3 isolate PD715 was a weak pathogen on oilseed rape, whereas PD589 caused severe disease symptoms in Quartz (Figs. 1 and 2). Intriguingly, the biomass reductions of PD589 in Quartz were in contrast to the symptoms on Incentive that, despite extensive stem colonization, tolerated infection by PD589 (Figs. 1, 2, and 3).
Virulence of Verticillium isolates is generally associated with successful host colonization (de Jonge et al. 2013). For example, in the production of effector proteins that are important for virulence colonizes, $V$. dahliae impaired its host plant to a lesser extent than the wild-type strain. Accordingly, $V$. longisporum colonizes more susceptible oilseed rape cultivars more successfully than less susceptible cultivars (Knüfer et al. 2017). In this study, the weak pathogens PD356 and PD715 were generally not detected in the host stem, which can explain the weak symptom development (Figs. 1, 2, and 3). Furthermore, the extent of colonization in the stem was negatively correlated with the aboveground biomass of Quartz and Clapton. The correlation for Incentive was not significant and, in Hilton, no significant differences in fungal colonization were observed between isolates. The lack of correlation in Incentive may be caused by the lower biomass reduction upon infection. This can be due to higher resistance of Incentive to $V$. longisporum when compared with the other Brassica cultivars. However, the contribution of different
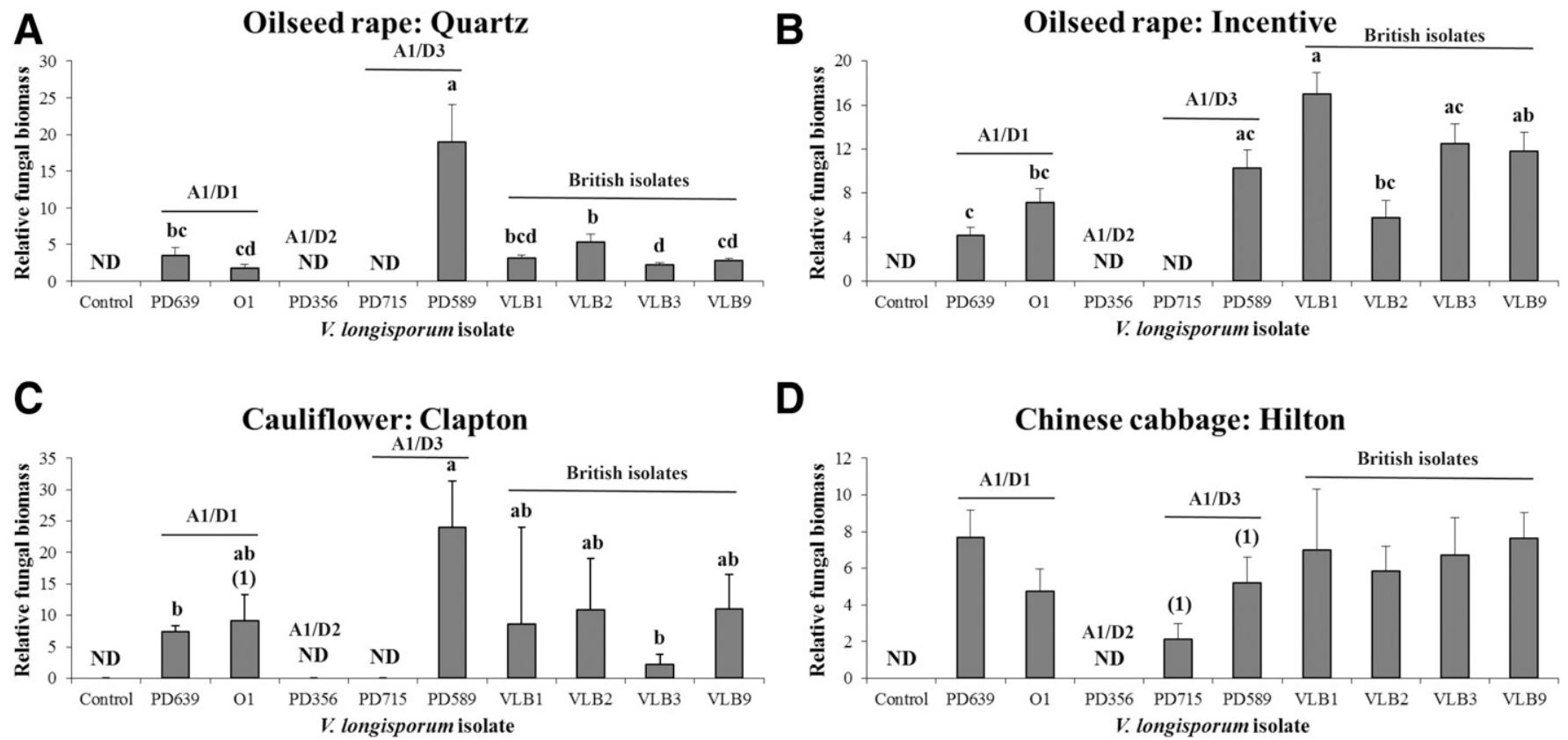

D

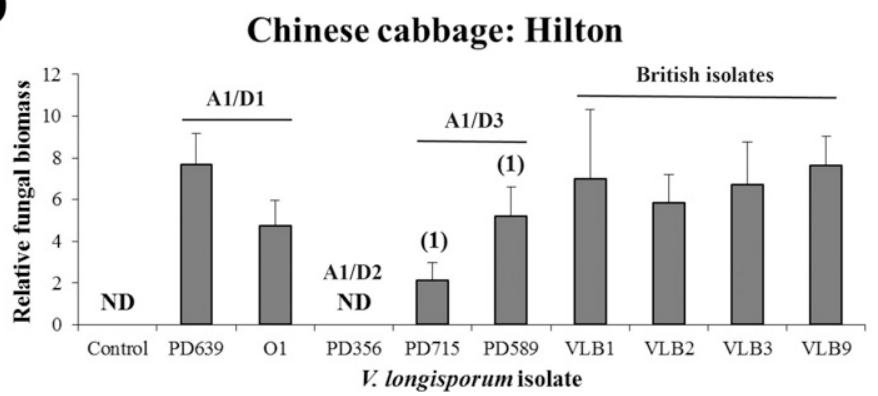

Fig. 3. Fungal biomass accumulation of various Verticillium longisporum strains in plant stems of cultivars A, Quartz; B, Incentive; C, Clapton; and D, Hilton. Bars indicate the median $V$. longisporum biomass relatively to the stem biomass. Isolates with no bar and ND in the graph were not detected in all five biological replicates. Significant differences were calculated with the Mann-Whitney $U$ test $(P<0.05)$ and depicted by different letter labels. No significant differences in colonization between isolates were found in Hilton Chinese cabbage. Numbers in parentheses indicate the number of samples without detection. No number is given if the fungal colonization in all replicates was detected. Error flags represent the standard error.

\section{Arabidopsis: Col-0}

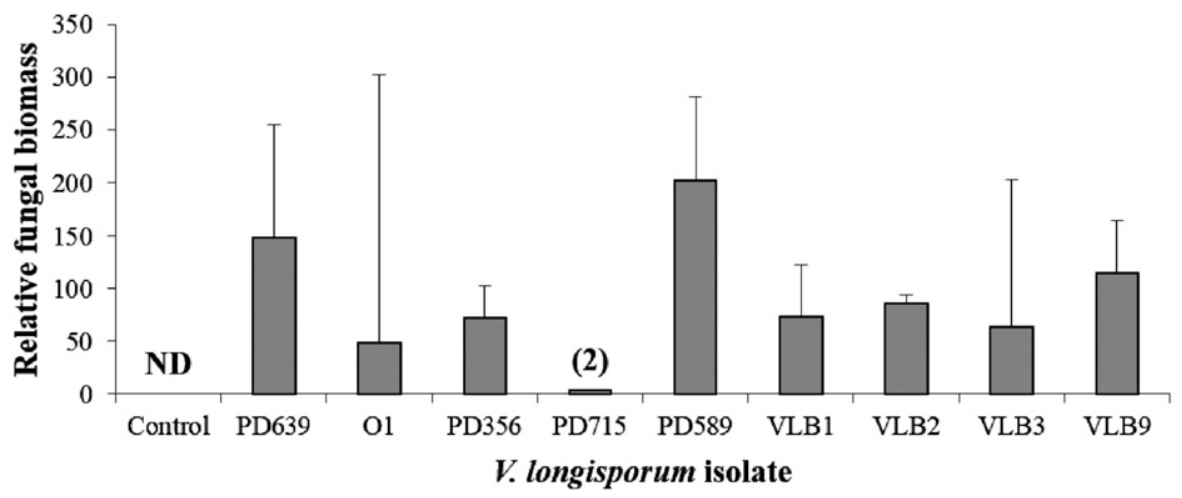

Fig. 4. Fungal biomass accumulation of various Verticillium longisporum strains in Arabidopsis plants. Bars indicate the median V. longisporum biomass relatively to plant biomass. Isolates with no bar and ND in the graph were not detected in all five biological replicates. No significant differences in colonization of the different isolates were found (Mann-Whitney $U$ test, $P<0.05$ ). The number in parentheses indicates the number of samples without detection. No number is given if the fungal colonization in all replicates was detected. Error flags represent the standard error. 
environmental conditions to the different disease responses cannot be excluded, because pathogenicity tests were performed at different time points. Remarkably, although A1/D1 isolates and PD589 caused similar disease symptoms, PD589 clearly colonized Quartz plants better than A1/ D1 isolates (Figs. 2A and 3A). Moreover, PD589 colonized Incentive to a similar extant as A1/D1 isolates but neither caused significant vascular discoloration or plant biomass reduction (Figs. 2B and 3B). Thus, fungal colonization may be a misleading indicator for virulence comparison, because hosts may tolerate particular pathogen strains better than others. Interspecific hybridization is an intrusive evolutionary mechanism because hybrid organisms experience a so-called "genomic shock" that incites major genomic rearrangement and changes in gene expression patterns (Doyle et al. 2008). Conceivably, hybridization may have affected the $V$. longisporum lineages differently, which may have led to different pathogenic features between lineages. However, more studies that investigate colonization patterns must be performed in order to relate colonization patterns to specific $V$. longisporum lineages more thoroughly.

Hybrid pathogens can have devastating outcomes on ecosystems. For example, the hybrid pathogen Phytophthora $\times$ alni led to an epidemic of alder decline in riparian ecosystems of Europe beginning in the early 1990s (Brasier et al. 1995). The hybrid fungus $V$. longisporum is currently gaining momentum because it is causing Verticillium stem striping in previously unaffected regions (CFIA 2015; Gladders et al. 2011). A decade since the first report of Verticillium stem striping in the United Kingdom, the extent to which this disease may contribute to losses in British oilseed rape is better understood. Although tested on a limited number of isolates (yet adequately representing the general genotypic diversity), the British V. longisporum population consists of strong pathogens that are as virulent as a previously characterized A1/D1 strain from oilseed rape. Therefore, given the similar climatic constraints, Verticillium stem striping is expected to have outcomes similar to those in countries where the disease was previously established, such as Germany. However, the British V. longisporum population is genotypically more diverse than the ones in Germany and Sweden (Depotter et al. in press). Conceivably, therefore, the heterogeneous character of the British populations may hamper disease management strategies more. Nevertheless, these recent findings should be an incentive in oilseed rape breeding programs to select for Verticillium stem striping resistance, especially because protective or curative control by conventional fungicides is not possible for Verticillium diseases.

\section{ACKNOWLEDGMENTS}

We thank the Marie Curie Actions program of the European Commission that financially supports the research investigating the threat of V. longisporum to U.K. oilseed rape production. Work in the laboratory of B. P. H. J. Thomma is supported by the Research Council Earth and Life Sciences of the Netherlands Organization of Scientific Research.

\section{LITERATURE CITED}

Anderson, P. K., Cunningham, A. A., Patel, N. G., Morales, F. J., Epstein, P. R., and Daszak, P. 2004. Emerging infectious diseases of plants: Pathogen pollution, climate change and agrotechnology drivers. Trends Ecol. Evol. 19:535-544.

Brasier, C. M., Rose, J., and Gibbs, J. N. 1995. An unusual Phytophthora associated with widespread alder mortality in Britain. Plant Pathol. 44: 999-1007.

CFIA. 2015. Verticillium wilt-Verticillium longisporum. Online publication. Canadian Food Inspection Agency. http://www.inspection.gc.ca/plants/ plant-pests-invasive-species/diseases/verticillim-wilt/eng/1420746212959/ 1420746213803

Debode, J., Van Poucke, K., França, S. C., Maes, M., Höfte, M., and Heungens, K. 2011. Detection of multiple Verticillium species in soil using density flotation and real-time polymerase chain reaction. Plant Dis. 95: 1571-1580.

de Jonge, R., Bolton, M. D., Kombrink, A., van den Berg, G. C., Yadeta, K. A., and Thomma, B. P. H. J. 2013. Extensive chromosomal reshuffling drives evolution of virulence in an asexual pathogen. Genome Res. 23: 1271-1282.

Depotter, J. R. L., Deketelaere, S., Inderbitzin, P., Von Tiedemann, A., Höfte, M., Subbarao, K. V., Wood, T. A., and Thomma, B. P. H. J. 2016a. Verticillium longisporum, the invisible threat to oilseed rape and other brassicaceous plant hosts. Mol. Plant Pathol. 17:1004-1016.

Depotter, J. R. L., Seidl, M. F., Wood, T. A., and Thomma, B. P. H. J. 2016 b. Interspecific hybridization impacts host range and pathogenicity of filamentous microbes. Curr. Opin. Microbiol. 32:7-13.

Depotter, J. R. L., Seidl, M. F., van den Berg, G. C. M., Thomma, B. P. H. J., and Wood, T. A. A distinct and genetically diverse lineage of the hybrid fungal pathogen Verticillium longisporum population causes stem striping in British oilseed rape. Environ. Microbiol. In press. doi:10.1111/14622920.13801

Doyle, J. J., Flagel, L. E., Paterson, A. H., Rapp, R. A., Soltis, D. E., Soltis, P. S., and Wendel, J. F. 2008. Evolutionary genetics of genome merger and doubling in plants. Annu. Rev. Genet. 42:443-461.

Ellendorff, U., Fradin, E. F., de Jonge, R., and Thomma, B. P. H. J. 2009. RNA silencing is required for Arabidopsis defense against Verticillium wilt disease. J. Exp. Bot. 60:591-602.

Eynck, C., Koopmann, B., Grunewaldt-Stoecker, G., Karlovsky, P., and von Tiedemann, A. 2007. Differential interactions of Verticillium longisporum and V. dahliae with Brassica napus detected with molecular and histological techniques. Eur. J. Plant Pathol. 118:259-274.

Eynck, C., Koopmann, B., and von Tiedemann, A. 2009. Identification of Brassica accessions with enhanced resistance to Verticillium longisporum under controlled and field conditions. J. Plant Dis. Prot. 116:63-72.

Floerl, S., Druebert, C., Majcherczyk, A., Karlovsky, P., Kües, U., and Polle, A. 2008. Defence reactions in the apoplastic proteome of oilseed rape (Brassica napus var. napus) attenuate Verticillium longisporum growth but not disease symptoms. BMC Plant Biol. 8:129.

Fradin, E. F., and Thomma, B. P. H. J. 2006. Physiology and molecular aspects of Verticillium wilt diseases caused by $V$. dahliae and V. albo-atrum. Mol. Plant Pathol. 7:71-86.

Fulton, T. M., Chunwongse, J., and Tanksley, S. D. 1995. Microprep protocol for extraction of DNA from tomato and other herbaceous plants. Plant Mol. Biol. Rep. 13:207-209.

Gladders, P., Ritchie, F., Barbara, D., Clarkson, J., Chantry, T., Taylor, M., and Turner, J. 2013. Importance and management of Verticillium wilt in winter oilseed rape. HGCA Project Report 512. Online publication. AHDB. https:// cereals.ahdb.org.uk/publications/2013/august/22/importance-and-managementof-verticillium-wilt-in-winter-oilseed-rape.aspx

Gladders, P., Smith, J. A., Kirkpatrick, L., Clewes, E., Grant, C., Barbara, D., Barnes, A. V., and Lane, C. R. 2011. First record of Verticillium wilt (Verticillium longisporum) in winter oilseed rape in the UK. New Dis. Rep. 23:8.

Heale, J. B., and Karapapa, V. K. 1999. The Verticillium threat to Canada's major oilseed crop: Canola. Can. J. Plant Pathol. 21:1-7.

Inderbitzin, P., Davis, R. M., Bostock, R. M., and Subbarao, K. V. 2011. The ascomycete Verticillium longisporum is a hybrid and a plant pathogen with an expanded host range. PLoS One 6:e18260.

Inderbitzin, P., and Subbarao, K. V. 2014. Verticillium systematics and evolution: How confusion impedes Verticillium wilt management and how to resolve it. Phytopathology 104:564-574.

Isaac, I. 1957. Verticillium wilt of Brussels sprout. Ann. Appl. Biol. 45: 276-283.

Karapapa, V. K., Bainbridge, B. W., and Heale, J. B. 1997. Morphological and molecular characterization of Verticillium longisporum comb. nov., pathogenic to oilseed rape. Mycol. Res. 101:1281-1294.

Knüfer, J., Lopisso, D. T., Koopmann, B., Karlovsky, P., and von Tiedemann, A. 2017. Assessment of latent infection with Verticillium longisporum in field-grown oilseed rape by qPCR. Eur. J. Plant Pathol. 147:819-831.

Kroeker, G. 1970. Vissnesjuka på rabs och rybs i Skåne orsakad av Verticillium [Verticillium on oilseed rape and turnip rape in Scania caused by Verticillium]. Svensk Frötidning 19:10-13.

Levin, A. G., Lavee, S., and Tsror, L. 2003. Epidemiology of Verticillium dahliae on olive (cv. Picual) and its effect on yield under saline conditions. Plant Pathol. 52:212-218.

Melero-Vara, J. M., Blanco-López, M. A., Bejarano-Alcázar, J., and Jiménez-Díaz, R. M. 1995. Control of Verticillium wilt of cotton by means of soil solarization and tolerant cultivars in southern Spain. Plant Pathol. 44: 250-260.

Novakazi, F., Inderbitzin, P., Sandoya, G., Hayes, R. J., von Tiedemann, A., and Subbarao, K. V. 2015. The three lineages of the diploid hybrid Verticillium longisporum differ in virulence and pathogenicity. Phytopathology 105:662-673.

R Core Team 2015. R: A Language and Environment for Statistical Computing. R Foundation for Statistical Computing, Vienna. https://www.Rproject.org/ 
Steventon, L. A., Fahleson, J., Hu, Q., and Dixelius, C. 2002. Identification of the causal agent of Verticillium wilt of winter oilseed rape in Sweden, V. longisporum. Mycol. Res. 106:570-578.

Tyvaert, L., França, S. C., Debode, J., and Höfte, M. 2014. The endophyte Verticillium Vt305 protects cauliflower against Verticillium wilt. J. Appl. Microbiol. 116:1563-1571.

Yu, J. M., Cafarov, I. H., and Babadoost, M. 2016. Morphology, molecular identity, and pathogenicity of Verticillium dahliae and V. longisporum associated with internally discolored horseradish roots. Plant Dis. 100: $749-757$

Zeise, K., and von Tiedemann, A. 2002. Host specialization among vegetative compatibility groups of Verticillium dahliae in relation to Verticillium longisporum. J. Phytopathol. 150:112-119.

Zhou, L., Hu, Q., Johansson, A., and Dixelius, C. 2006. Verticillium longisporum and $V$. dahliae: Infection and disease in Brassica napus. Plant Pathol. 55:137-144. 